Radiocarbon, Vol 25, No 3, 1983, P 867-874]

\title{
INSTITUT ROYAL DU PATRIMOINE ARTISTIQUE \\ RADIOCARBON DATES IX
}

\section{MICHĖLE DAUCHOT-DEHON, MARK VAN STRYDONCK, and} JOS HEYLEN

Institut Royal du Patrimoine Artistique, Koninklijk Instituut voor het

Kunstpatrimonium, Brussels, Belgium

This list contains the results of ${ }^{14} \mathrm{C}$ age determinations obtained at the laboratory in 1981-1982. Samples are analyzed in three new proportional counters which are described in R, 1980, v 22, p 442. Our installation differs from that of Heidelberg in that our filling gas is methane.

\section{ACKNOWLEDGMENTS}

The authors would like to thank the ${ }^{14} \mathrm{C}$ laboratory in Lyon and in Groningen for $\delta^{13} \mathrm{C}$ measurements. The assistance of M Dupas in analyzing mortar samples is gratefully acknowledged.

\section{IRPA-411. Zoniënbos}

I. GEOLOGIG SAMPLES

\section{A. Belgium}

Wood (Fagus) from Zoniënbos in Brabant ( $50^{\circ} 48^{\prime} 14^{\prime \prime} \mathrm{N}, 4^{\circ} 48^{\prime} 17^{\prime \prime}$ E) at $125 \mathrm{~cm}$ below surface. Coll Dec 1980 and subm Jan 1981 by $\mathrm{R}$ Langohr, Univ Gent, Belgium. Comment: expected date 10,000-13,000 вР (Langohr, 1981).

\section{IRPA-452. De Haan}

$2470 \pm 50$

Shells from sand dunes at De Haan in W Vlaanderen $\left(58^{\circ} 18^{\prime} \mathrm{N}, 3^{\circ}\right.$ $05^{\prime}$ E) at 130cm below surface. Coll Aug 1982 and subm Sept 1982 by F Verhaeghe, Univ Gent. Comment: result used to date sand dune formation in coastal plain; agrees with expected age.

\section{Ipenrooi series}

Material from peat bog sec at Ipenrooi, Antwerpen $\left(51^{\circ} 28^{\prime} 40^{\prime \prime} \mathrm{N}\right.$, $4^{\circ} 45^{\prime} 04^{\prime \prime} \mathrm{E}$ ). Coll and subm 1980 by L Beyens, Univ Antwerpen, Belgium. In $75 \mathrm{~cm}$ core, pollen was taken every $2 \mathrm{~cm}$.

IRPA-391. Ipenrooi-ven 1

$$
7350 \pm 120
$$

Peat from 75 to $74 \mathrm{~cm}$. Atlantic age expected.

\section{IRPA-472. Ipenrooi-ven 2}

Peat from 73 to $72 \mathrm{~cm}$. Atlantic age expected.

IRPA-392. Ipenrooi-ven 3

Peat from 60 to $56 \mathrm{~cm}$. Sub-boreal age expected.

IRPA-393. Ipenrooi-ven 4

$1160 \pm 100$

Peat from 52 to $48 \mathrm{~cm}$. Sub-atlantic age expected.

IRPA-394. Ipenrooi-ven 5

$$
260 \pm 80
$$

Peat from 42 to $37 \mathrm{~cm}$. Sub-atlantic age expected. 
868 Michèle Dauchot-Dehon, Mark Van Strydonck, and Jos Heylen

IRPA-404. Ipenrooi-ven B

$3105 \pm 90$

Peat from 56 to $48 \mathrm{~cm}$ from profile similar to IRPA-392. Sub-boreal age expected.

General Comment: dates agree with pollen analysis (Beyens, 1982).

IRPA-403. Meerle

$9020 \pm 120$

Peat from bog sec at Meerle, Antwerpen $\left(51^{\circ} 28^{\prime} 50^{\prime \prime} \mathrm{N}, 4^{\circ} 46^{\prime} 49^{\prime \prime}\right.$ E). Coll and subm 1980 by L Beyens. In core of $88 \mathrm{~cm}$, many pollen samples and one for ${ }^{14} \mathrm{C}$ dating were taken from 85 to $83 \mathrm{~cm}$. This profile is related to Ipenrooi series. Comment (LB): date is perhaps too young; expected date: 9660 BP (Beyens, 1982).

\section{Bredene series}

Peat from coastal plain at Bredene in W Vlaanderen $\left(51^{\circ} 14^{\prime} 20^{\prime \prime} \mathrm{N}\right.$, $2^{\circ} 57^{\prime} 30^{\prime \prime} \mathrm{E}$ ). Coll and subm 1981 by C Verbruggen, Univ Gent. Dating results and pollen analysis indicate beginning and end of peat growth.

IRPA-437. Sample 1

Base of peat layer.

IRPA-438. Sample 2

Top of peat layer.

IRPA-439. Adinkerke 193DB5M53 $\left(51^{\circ} 04^{\prime} 30^{\prime \prime} \mathrm{N}, 2^{\circ} 35^{\prime} 15^{\prime \prime} \mathrm{E}\right)$. Coll and subm 1981 by C Verbruggen. Comment: dates agree well with beginning of Atlantic.

\section{Dunes series}

Peat from coastal plain in W Vlaanderen. Coll Oct 1980 by $R$ De Ceunynck and M Van Strydonck and subm Oct 1980 by R De Ceunynck, Univ Gent. Results are used to study stratigraphy of sand dunes.

IRPA-405. De Panne AC 37

$3090 \pm 80$

Base of peat layer $\left(51^{\circ} 04^{\prime} 02^{\prime \prime} \mathrm{N}, 2^{\circ} 34^{\prime} 47^{\prime \prime} \mathrm{E}\right)$.

IRPA-436. Nieuwmunster $48 H B 10$ bis

$440 \pm 70$

Peat from layer at $183 \mathrm{~cm}$ below surface $\left(51^{\circ} 17^{\prime} 29^{\prime \prime} \mathrm{N}, 3^{\circ} 03^{\prime} 45^{\prime \prime} \mathrm{E}\right)$.

IRPA-447. De Panne DW 5A

Modern E).

Wood from sand layer, 110 to $180 \mathrm{~cm}$ below surface $\left(51^{\circ} 04^{\prime} \mathrm{N}, 2^{\circ} 34^{\prime}\right.$

IRPA-418. De Panne DW 5B

Modern

Soil with sand, 180 to $195 \mathrm{~cm}$ below surface $\left(51^{\circ} 04^{\prime} \mathrm{N}, 2^{\circ} 34^{\prime} \mathrm{E}\right)$.

IRPA.449. De Panne DW 5C

$1310 \pm 70$

Peat from layer, 225 to $255 \mathrm{~cm}$ below surface $\left(51^{\circ} 04^{\prime} \mathrm{N}, 2^{\circ} 34^{\prime} \mathrm{E}\right)$.

Mark series

The following results complete previously pub list $(\mathrm{R}, 1981, \mathrm{v} 23, \mathrm{P}$ 
Institut Royal du Patrimoine Artistique Radiocarbon Dates IX 869 345-346) of samples from alluvial plain of Mark R in W Vlaanderen, Hainaut and Brabant. Coll and subm 1981-1982 by W Huybrechts, Geol Inst, Free Univ Brussels.

IRPA-440. Lessines B 80/4/3(1) $\quad 5800 \pm 90$ E).

Clayey peat from layer, 260 to $290 \mathrm{~cm}$ below surface $\left(50^{\circ} 44^{\prime} \mathrm{N}, 3^{\circ} 53^{\prime}\right.$

IRPA-441. Lessines B 80/4/3(2) $\quad 8370 \pm 120$

Peat with vegetable residues, 405 to $440 \mathrm{~cm}$ below surface $\left(50^{\circ} 44^{\prime} \mathrm{N}\right.$, $\left.3^{\circ} 53^{\prime} \mathrm{E}\right)$.

IRPA-442. Galmaarden B 80/8/27 $1730 \pm 80$ E).

Wood from sand layer, 180 to $200 \mathrm{~cm}$ below surface $\left(50^{\circ} 45^{\prime} \mathrm{N}, 3^{\circ} 57^{\prime}\right.$

IRPA-443. Moerbeke B 80/2/12(2) $\quad 6710 \pm 100$ $\left.3^{\circ} 55^{\prime} \mathrm{E}\right)$

Peat with vegetable residues, 330 to $355 \mathrm{~cm}$ below surface $\left(50^{\circ} 45^{\prime} \mathrm{N}\right.$,

IRPA-444. Moerbeke B 80/2/12(3)

$8470 \pm 120$

Peat from layer, 390 to $430 \mathrm{~cm}$ below surface $\left(50^{\circ} 45^{\prime} \mathrm{N}, 3^{\circ} 55^{\prime} \mathrm{E}\right)$.

IRPA-445. Moerbeke B 80/2/12(1) $8000 \pm 90$

Clayey peat with pieces of wood, 355 to $390 \mathrm{~cm}$ below surface $\left(50^{\circ} 45^{\prime}\right.$ $\left.\mathrm{N}, 3^{\circ} 55^{\prime} \mathrm{E}\right)$.

IRPA-473. Enghien B 81/6/19 $1700 \pm 70$

Peat from layer, 110 to $125 \mathrm{~cm}$ below surface $\left(50^{\circ} 41^{\prime} \mathrm{N}, 4^{\circ} 00^{\prime} 30^{\prime \prime} \mathrm{E}\right)$.

IRPA-481. Galmaarden B 80/6/37(1) $\quad 8700 \pm 110$

Peat from layer, 585 to $595 \mathrm{~cm}$ below surface $\left(50^{\circ} 45^{\prime} \mathrm{N}, 3^{\circ} 37^{\prime} \mathrm{E}\right)$.

IRPA-482. Galmaarden B 80/6/37(2) $\quad 6490 \pm 100$

Clay from layer, 606 to $614 \mathrm{~cm}$ below surface $\left(50^{\circ} 45^{\prime} \mathrm{N}, 3^{\circ} 37^{\prime} \mathrm{E}\right)$.

IRPA-484. Galmaarden B 82/6/16(2) $9090 \pm 110$ $\left.37^{\prime} \mathrm{E}\right)$

Peat with shell residues, 620 to $630 \mathrm{~cm}$ below surface $\left(50^{\circ} 45^{\prime} \mathrm{N}, 3^{\circ}\right.$

IRPA-485. Marcq B 82/6/41 $990 \pm 80$ E).

Clayey peat from layer, 290 to $300 \mathrm{~cm}$ below surface $\left(50^{\circ} 40^{\prime} \mathrm{N}, 3^{\circ} 59^{\prime}\right.$

General Comment (WH): dates confirm previous results and describe continuous peat growth in alluvial plain, beginning ca 9000 BP, ending ca 5500 вр. In parts of basin upstream, peat layer is much younger, ca 2000 to $1000 \mathrm{BP}$.

IRPA-501. Murama

B. Africa

$160 \pm 70$

Charcoal at alt of Murama, Bujumbura $\left(2^{\circ} 33^{\prime} \mathrm{N}, 2^{\circ} \mathrm{E}\right)$, from low 
terrace of Ruzizi $\mathrm{R}$ at $300 \mathrm{~cm}$ above lake Tanganika. Coll by L Ilunga, Free Univ Brussels, and subm Oct 1980 by C Baeteman. Comment: young date can be explained by hydrography of area.

\section{Taourga series}

Organic material and calcareous crust from Taourga, Algeria $\left(36^{\circ}\right.$ $10^{\prime} 12^{\prime \prime} \mathrm{N}, 3^{\circ} 05^{\prime} 17^{\prime \prime} \mathrm{E}$ ). Coll and subm Feb 1981 by L Bock, Fac Agronom Gembloux, Belgium. Comment: dates are used in morphol and pedol study in which soils and calcareous crusts are assoc (Bock and Mathieu, 1982).

IRPA-429. 2.5

$5210 \pm 260$

Organic material extracted from vertisol.

IRPA-430. 24.3

$3820 \pm 210$

Organic material extracted from red soil, diluted, $27 \%$ sample.

IRPA-431. IV

Calcareous crust from basin.

$10,940 \pm 140$

IRPA-432. $\quad C_{3}$

$24,650 \pm 620$

Calcareous crust from $\mathrm{E}$ border of upland.

IRPA-433. I $_{3}$

Calcareous crust from border of upland.

$34,080 \pm 1940$

\section{ARCHAEOLOGIC SAMPLES}

\section{St-Lambert series}

\section{A. Belgium}

The following results complete previously pub list ( $\mathrm{R}, 1981, \mathrm{v} 23, \mathrm{p}$ 349-350) from archaeol excavation at Place St-Lambert, Liège $\left(50^{\circ} 38^{\prime} 45^{\prime \prime}\right.$ N, $5^{\circ} 34^{\prime} 30^{\prime \prime}$ E). Coll Aug 1980 by M Van Strydonck and subm Aug 1980 by J Alénus-Lecerf, Nat Service Excavations, Belgium.

TABLE 1

St-Lambert radiocarbon dates

\begin{tabular}{|c|c|c|c|c|c|}
\hline $\begin{array}{l}\text { IRPA } \\
\text { no. }\end{array}$ & $\begin{array}{c}\text { Ref } \\
\text { (Sample no.) }\end{array}$ & Material & $\begin{array}{l}\text { Depth } \\
(\mathrm{cm})\end{array}$ & ${ }^{14} \mathrm{C}$ age & $\begin{array}{c}\text { Expected age } \\
\text { (AD) }\end{array}$ \\
\hline-395 & 7 & Wood & $\begin{array}{c}200 \\
\text { under wall }\end{array}$ & $1010 \pm 30$ & $<1185$ \\
\hline-396 & 7 & Mortar & Street level & $\begin{array}{c}2130 \pm 50 \\
\delta^{13} \mathrm{C}=-15.8 \%\end{array}$ & $<1185$ \\
\hline$-397^{\text {bis }}$ & 6 & Mortar & $\begin{array}{l}300 \text { to } 400 \\
\text { under wall }\end{array}$ & $\begin{array}{c}1660 \pm 40 \\
\delta^{13} \mathrm{C}=-12.8 \%\end{array}$ & $>1185$ \\
\hline-398 & 6 & Mortar & Street level & $\begin{array}{c}2700 \pm 50 \\
\delta^{13} \mathrm{C}=-12.1 \%\end{array}$ & $>1185$ \\
\hline-399 & 7 & Mortar & $\begin{array}{c}200 \\
\text { under wall }\end{array}$ & $\begin{array}{c}1060 \pm 50 \\
\delta^{13} \mathrm{C}=-17.0 \%\end{array}$ & $<1185$ \\
\hline-400 & 2 & Mortar & Street level & $\begin{array}{c}1290 \pm 50 \\
\delta^{13} \mathrm{C}=-12.4 \%\end{array}$ & $>1185$ \\
\hline-401 & 6 & Wood (pile) & Under wall & $800 \pm 70$ & \\
\hline$-40 ?$ & 6 & Wood (pile) & Under wall & $550 \pm 70$ & \\
\hline-479 & & Charcoal & & $740 \pm 70$ & \\
\hline-480 & & Wood (pile) & & $490 \pm 110$ & \\
\hline
\end{tabular}


General Comment: charcoal and wood yielded dates that agree well; mortar carbonate gave old ages probably caused by infiltration from several floods (Van Strydonck, Dupas, and Dauchot-Dehon, 1982).

\section{Antwerpen series}

Mortar from "Onze-Lieve-Vrouw" cathedral and from three houses in Antwerpen ( $\left.51^{\circ} 13^{\prime} 16^{\prime \prime} \mathrm{N}, 4^{\circ} 23^{\prime} 60^{\prime \prime} \mathrm{E}\right)$. Coll 1981 by M Van Strydonck, A De Nayer, and R Tijs, Dienst Monumentenzorg Antwerpen, and subm 1981 by M Van Strydonck. Samples were taken from pillars of cathedral at 150 to $600 \mathrm{~cm}$ above street level and from floors or walls of houses.

TABLE 2

Antwerpen radiocarbon dates

\begin{tabular}{|c|c|c|c|c|c|}
\hline $\begin{array}{l}\text { IRPA } \\
\text { no. }\end{array}$ & Ref & Material & $\begin{array}{l}\text { Depth } \\
(\mathrm{cm})\end{array}$ & ${ }^{14} \mathrm{C}$ age & $\begin{array}{c}\text { Expected age } \\
\text { (AD) }\end{array}$ \\
\hline-412 & 3rd pillar $S$ & Mortar & $\begin{array}{l}150 \text { above } \\
\text { street level }\end{array}$ & $\begin{array}{c}610 \pm 30 \\
\delta^{13} \mathrm{C}=-15.4 \%\end{array}$ & $1420-1435$ \\
\hline-413 & 6 th pillar $S$ & Mortar & $\begin{array}{l}180 \text { above } \\
\text { street level }\end{array}$ & $\begin{array}{c}580 \pm 30 \\
\delta^{13} \mathrm{C}=-14.3 \%\end{array}$ & $1420-1435$ \\
\hline-414 & 3rd pillar $\mathrm{N}$ & Mortar & $\begin{array}{l}250-300 \text { above } \\
\text { street level }\end{array}$ & $\begin{array}{c}410 \pm 30 \\
\delta^{13} \mathrm{C}=-13.8 \%\end{array}$ & $1420-1435$ \\
\hline-415 & 5 th pillar $\mathrm{N}$ & Mortar & $\begin{array}{l}600 \text { above } \\
\text { street level }\end{array}$ & $\begin{array}{c}760 \pm 30 \\
\delta^{13} \mathrm{C}=-16.0 \%\end{array}$ & $1420-1435$ \\
\hline-418 & & Mortar & $\begin{array}{l}\text { Foot of } \\
\text { pillar }\end{array}$ & $\begin{array}{c}\text { Modern } \\
\delta^{13} \mathrm{C}=-19.4 \%\end{array}$ & $1420-1435$ \\
\hline-416 & $\begin{array}{l}\text { House } \\
\text { Rodenborg }\end{array}$ & Mortar & $\begin{array}{l}15-20 \text { under } \\
\text { floor }\end{array}$ & $\begin{array}{c}\text { Modern } \\
\delta^{13} \mathrm{C}=-9.3 \%\end{array}$ & 1550 \\
\hline-420 & $\begin{array}{l}\text { House on } \\
\text { "Grooten } \\
\text { Helm" ground }\end{array}$ & Mortar* & In wall & $\begin{array}{c}840 \pm 90 \\
\delta^{13} \mathrm{C}=-9.7 \%\end{array}$ & 1500 \\
\hline-421 & $\begin{array}{l}\text { "Jacob Jor- } \\
\text { daens" House }\end{array}$ & Mortar* & In wall & $\begin{array}{c}1120 \pm 80 \\
\delta^{13} \mathrm{C}=-11.9 \%\end{array}$ & 1640 \\
\hline
\end{tabular}

General Comment: mortar samples were first examined to separate fractions containing chalk carbonate from those containing carbonate formed after mortar preparation (Van Strydonck, Dupas, and Dauchot-Dehon, 1982). Dates for pillar of cathedral agree with expected ages. Mortars with $\left({ }^{*}\right)$ are diluted, $80 \%$ sample.

\section{Vrasene series}

Mortar and charcoal from Vrasene in O Vlaanderen $\left(51^{\circ} 13^{\prime} \mathrm{N}, 4^{\circ}\right.$ 12' E). Coll and subm Aug 1980 by R Van Hove.

IRPA-422. Vr K80/II/5, 6/A

$$
940 \pm 90
$$

Charcoal from $115 \mathrm{~cm}$ below street level.

IRPA-424. Sleuf 80/I

$$
1090 \pm 120
$$

Mortar from foot of pillar of Romanesque church. Comment: diluted, $48.5 \%$ sample.

\section{IRPA-425. Sleuf 80/II}

Mortar from foot of pillar of Romanesque church. 
872 Michèle Dauchot-Dehon, Mark Van Strydonck, and Jos Heylen

IRPA-426. Vr K80/II/ $\mathbf{C}_{2}$

$820 \pm 70$

Charcoal from top of leveling layer under oldest floor level.

General Comment (RVH): IRPA-422 and -426 corroborated archaeol and stratigraphic data. IRPA-425 is too old.

\section{Gent series}

Wood from Gent in $\mathrm{O}$ Vlaanderen $\left(51^{\circ} 06^{\prime} \mathrm{N}, 3^{\circ} 45^{\prime} \mathrm{E}\right)$ at 100 to $200 \mathrm{~cm}$ below street level. Coll and subm 1980 by V Van Doorne, Dienst Monumentenzorg Gent.

IRPA-409. Profile B-C

$$
1260 \pm 70
$$

IRPA-410. Profile A-D

$$
\mathbf{3 3 0} \pm \mathbf{8 0}
$$

General Comment: archaeol date: 12th-14th centuries AD.

\section{Flobecq series}

Samples from Flobecq, Hainaut $\left(50^{\circ} 44^{\prime} 50^{\prime \prime} \mathrm{N}, 3^{\circ} 43^{\prime} 34^{\prime \prime} \mathrm{E}\right)$. Coll and subm by A Roolant, Fed archéol Wallonie. Samples are stratigraphically defined (Faider-Feytmans, 1980).

IRPA-427. Sample 1 $1910 \pm 130$

Peat from layer in Roman well. Comment: diluted, $40.16 \%$ sample.

IRPA-428. Sample 2

Wood from Roman well.

$2000 \pm 80$

IRPA-446. Waasmunster

$1940 \pm 60$

Wood from Roman well in Waasmunster, O Vlaanderen $\left(51^{\circ} 06^{\prime} 25^{\prime \prime}\right.$ N, $4^{\circ} 05^{\prime} 12^{\prime \prime}$ E). Coll and subm Aug 1981 by C Verbruggen. Comment: result agrees with archaeol date: 1st century AD.

\section{Wortegem-Petegem series}

Charcoal from "Oud Kasteel" site along Schelde R at WortegemPetegem, O Vlaanderen (50 50 01" N, $\left.3^{\circ} 33^{\prime} 19^{\prime \prime} \mathrm{E}\right)$. Coll and subm 1982 by D Callebaut, Nat Service Excavations (Callebaut, 1981).

IRPA-474. Pe 77/1

$$
990 \pm 60
$$

Sample from furnace. Archaeol date: 9th-10th century AD.

IRPA-475. Pe 77/6

$1550 \pm 80$

Sample from fireplace in wooden house. This oldest building dates from 8th century $\mathrm{AD}$, following stratigraphic data.

\section{IRPA-478. KZ B2 III}

$560 \pm 60$

Charcoal from furnace in Abbey church at St-Gillis-Dendermonde, O Vlaanderen $\left(51^{\circ} 01^{\prime} 11^{\prime \prime} \mathrm{N}, 4^{\circ} 06^{\prime} 42^{\prime \prime} \mathrm{E}\right)$. Coll 1981 by A Stroobants, Oudheidkundige Kring van het Land Dendermonde and subm 1982 by D Callebaut. Comment: archaeol date: AD 1228-1667.

IRPA-486. Webbekom $2260 \pm 70$

Grain of wheat from Webbekom, Brabant $\left(50^{\circ} 58^{\prime} 05^{\prime \prime} \mathrm{N}, 5^{\circ} 04^{\prime} 26^{\prime \prime}\right.$ 
E). Coll and subm 1980 by P Vermeersch, Univ Leuven. Comment: archaeol date: Iron age.

IRPA-495. WV 8219

$\mathbf{2 2 7 0} \pm \mathbf{7 0}$

Wood from calcined beam at Waudrez, Hainaut $\left(50^{\circ} 25^{\prime} 50^{\prime \prime} \mathrm{N}, 4^{\circ}\right.$ 09' 08" E). Coll and subm Sept 1982 by Ph Dekegel, Cercle archéol Waudrez. Comment: sample from Roman level; expected date: 1st-3rd century AD.

IRPA-500. A

$1185 \pm 50$

B

$1190 \pm 50$

Wood (Alnus) from plank in Veemarkt at Antwerpen $\left(51^{\circ} 13^{\prime} \mathrm{N}, 4^{\circ}\right.$ $23^{\prime}$ E) $250 \mathrm{~cm}$ under street level. Coll Jan 1982 by M Van Strydonck and T Oost, Oudheidkundige Mus Antwerpen; subm Jan 1982 by M Van Strydonck. Comment: expected date: 12th-13th century AD.

IRPA-453.

$940 \pm 50$

Wood from dugouts at Austruweel, Antwerpen $\left(51^{\circ} \mathrm{N}, 4^{\circ} \mathrm{E}\right)$. Coll 1910-1911 by Rahir, Mus Royaux Art Hist (Rahir, 1911; 1913); subm Sept 1982 by M Van Strydonck. Sample divided in fine parts and dated separately. Results are shown in table 3 which gives per mil depletion with regard to standard (Stuiver and Polach, 1977).

TABLE 3

Wood samples from Austruweel

\begin{tabular}{cc}
\hline IRPA-453. & $\mathrm{D}^{14} \mathrm{C} \%$ o \\
\hline 1 & -0.1026 \\
2 & -0.1153 \\
3 & -0.1014 \\
4 & -0.1184 \\
5 & -0.1133 \\
Mean & -0.1102 \\
\hline
\end{tabular}

General Comment (MVS): result shows that dugouts date from Middle ages and not from Iron age as expected (Ellmers, 1978; Van Strydonck, Dauchot-Dehon, and Heylen, in press). Dates are confirmed by Lv-826, $-827: 1050 \pm 65,820 \pm 45$ (Dauchot-Dehon et al, 1982).

IRPA-378. Br 79/1/19 $\quad 1920 \pm 70$

Peat from Roman site in Belgian coastal plain at Bredene $\left(51^{\circ} 14^{\prime}\right.$ $24^{\prime \prime} \mathrm{N}, 2^{\circ} 57^{\prime} 33^{\prime \prime} \mathrm{E}$ ). Coll and subm 1981 by $\mathrm{H}$ Thoen, Univ Gent. This sample completes previously pub list (R, 1981, v 23, p 348-349).

\section{Zadar series}

\section{B. Yugoslavia}

Mortar from St-Donat church in Zadar, Croatia $\left(44^{\circ} 06^{\prime} 48^{\prime \prime} \mathrm{N}, 15^{\circ}\right.$ $14^{\prime}$ 04" E). Subm Oct 1981 by D Srdoč, Inst "Rudjer Boškovic," Zagreb. The two samples were coll very carefully with precise description of loca- 
tions. Mortar samples were first examined to separate fraction containing chalk carbonate from those containing carbonate formed after mortar preparation.

\section{IRPA-498. Zadar 1}

\section{IRPA-499. Zadar 2}

$1610 \pm 70$ $\delta^{13} C=-12.1 \%$ o

$510 \pm 70$

$\delta^{1 s} C=-9.5 \%$

General Comment (DS): lime used to make mortars in Middle ages was prepared in primitive kilns where decomposition of limestone was not complete due to low firing temperature. Also, sand used for mortars was pure limestone, $i e$, dead carbonate. This may explain why date for IRPA498 is too old. More surprising is date for IRPA-499 that is too young, but we cannot exclude much younger mortar, since church was reconstructed, and destroyed, several times in history.

\section{REFERENCES}

Beyens, L, ms, 1982, Bydrage tot de holocene paleo-ecologie van het stroomgebied van de Mark in België, gebaseerd op de studie van diatomeeën, pollen en thecamoeba's: Thesis, Fac Sci, Univ Antwerpen.

Bock, $L$ and Mathieu, L, 1982, La genèse des accumulations calcaires vue sous l'angle de l'approche géomorpho-pédologique: Soc belge géol Bull, v 91, no. 1, p 19-26.

Callebaut, D, 1981, Het Oud Kasteel te Pelegem. I. De Karolingische curtis en haar ontwikkeling tot de XIIde eeuw: Archaeol Belgica, v 237, no. 8.

Dauchot-Dehon, M, Van Strydonck, M, Heylen, J, Gilot, E, Frix, F, and Devos, J, 1982, Dates carbone-14 concernant l'archéologie en Belgique: Helinium, v XXII, p 209. 237.

Ellmers, D, 1978, Shipping on the Rhine during the Roman period: The pictural evidence: CBA Research Rept, v 24, London, p 1-14.

Faider-Feytmans, G, 1980, Enseigne romaine découverte à Flobecq (Hainaut): Helinium, v XX, p 1-43.

Langohr, R, 1981, Correlatie tussen de morfologie van permafrostsporen en de landschapspositie in het Zoniënbos (Belgische Lössgebied): Nederlands-Belgische Palynol conf, 21st, Westerbork, Nederlands, Proc, p 26-28.

Rahir, E, 1911, Découverte d'une pirogue antique à Austruweel: Mus royale Arts décoratifs Bull, v 1, p 3-5.

1913, La deuxième pirogue protohistorique d'Austruweel: Mus royale Arts décoratifs Bull, v 1, p 3-6.

Stuiver, M and Polach, H A, 1977, Discussion: Reporting of ${ }^{14} \mathrm{C}$ data: Radiocarbon, v 19 , p 355-363.

Van Strydonck, M, Dauchot-Dehon, $\mathbf{M}$, and Heylen, J, in press, Radiokoolstofdatering van de Boomstamkano's van Austruweel: Inst Royal Patrimoine Artistique Bull, in press.

Van Strydonck, M, Dupas, M, and Dauchot-Dehon, M, in press, Radiocarbon dating of old mortars, in Mook, W $\mathrm{G}$ and $\mathrm{Waterbolk,} \mathrm{H} \mathrm{T}$, eds, Internatl symposium on ${ }^{14} \mathrm{C}$ and archaeol, 1st, Proc: Groningen, in press. 